\title{
A Hybrid Framework for Cloud Computing Adoption for Small and Medium Size Enterprises
}

\author{
Isaac K. Mwangi ${ }^{1}$, George O. Okeyo ${ }^{2}$, Michael W. Kimwele ${ }^{3}$ \\ ${ }^{1}$ Jomo Kenyatta University of Agriculture and Technology (JKUAT), Nairobi - KENYA isaacmwangi1@ gmail.com \\ ${ }^{2}$ Jomo Kenyatta University of Agriculture and Technology (JKUAT), Nairobi - KENYA gokeyo@jkuat.ac.ke \\ ${ }^{3}$ Jomo Kenyatta University of Agriculture and Technology (JKUAT), Nairobi - KENYA kimwele@icsit.jkuat.ac.ke
}

\begin{abstract}
Cloud Computing is a term that refers to resources and computer systems available on demand through the internet, which can provide a number of integrated computer services without being bound by local resources. Most SMEs are currently focusing on reducing their IT costs, looking for ways to make IT teams leaner and instead concentrating on remaining competitive in the market by putting more of their available resources into their core business as opposed to ICT operations. Therefore, for them to compete effectively, they will need IT infrastructure that can scale quickly to meet dynamic business demands and maximize utilization of their IT investments. Traditional IT systems have become more complex and costly to maintain and are no longer able to meet business demands since they lack flexibility and scalability. Cloud computing is the answer to the problems that are currently being experienced by SMEs. Cloud computing eliminates the need for high infrastructure expenses and also provides an easy to use, cost efficient, flexible, dynamic and secure environment for SMEs to conduct their business. Currently, there are inadequate guidelines for adopting cloud computing and building trust. Therefore, this paper develops a six-phase framework which provides SMEs with a roadmap for adopting cloud computing. The framework emphasizes on collaboration between subscribers and vendor, SME management involvement and adequate user support and training as they are essential for successful adoption of cloud computing.
\end{abstract}

Keywords: Cloud adoption, cloud computing, framework, security, SME

\section{INTRODUCTION}

According to specialists, cloud computing is one of the most significant transformation in information technology with many advantages to both companies and end users. This technology promises to release the client from the burden of administering more and more complex and expensive systems by offering them the possibility of using systems with state of the art computing capabilities, high availability and scalability [3]. Given that there is constant scrutiny over this subject, a plethora of definitions attempting to address the concept of cloud computing have emerged.
According to the national institute of technology and standards (NIST), cloud computing is a model of organizing computers for enabling convenient, ubiquitous, on-demand network access to a shared pool of configurable IT resources.

Cloud computing represents a fundamental change in how information technology is provisioned in that it enables computing facilities such as storage, computing power, network infrastructure and applications to be delivered as a method service over the internet [19]. There is evidence to suggest that cloud computing has become a strategic direction for many organizations and has the capacity to be employed in critical areas of an organisation's IT infrastructure. Cloud computing is a viable alternative mechanism to traditional ERP. Implementing cloud computing improves the traditional ERP in an organizational context. As the cloud computing paradigm continues to evolve, the benefits and risks associated with selecting a cloud solution became more understood and accepted by potential adopting organizations. Cloud computing adoption requires that organizations have readiness on multiple dimensions including: governance, process analysis and improvement, applications rationalization and modernization and hardware and software standardization.

\subsection{Small and medium size Enterprises (SMEs)}

It should be noted that a standard international definition of small and medium-sized enterprise does not exist [18]. SMEs are defined differently in the legislation across countries, in particular because the dimension "small" and "medium" of a firm are relative to the size of the domestic economy. SMEs play a major role in economic development in every country, including in African countries. Studies indicate that in both advanced economies and developing countries SMEs contribute on average 60 percent of total formal employment in the manufacturing sector [18]. For African economies, the contribution of the SME sector to job opportunities is even more important. Taking into account the contribution of the informal sector, SMEs account for about three-quarters of total employment in the manufacturing industry. Nevertheless, SMEs in Kenya are enterprises with fulltime employees not exceeding 100 or annual sales turnover not exceeding Kshs 150 
million [20]. Development of competitive and resilient SMEs forms an integral component of Kenya's initiatives to be a globally competitive and prosperous nation with a high quality of life by 2030 .

Global public-sector and SME cloud computing adoption remains more in the investigative stages than in actual deployments, whereas the private sectors seems more willing to invest in and deploy the technology [11]. While existing research on cloud computing has been undertaken from the service providers perspective there is need for further research that focuses on the organizational perspective [4]. A multiplicity of interpretations was confirmed in a 2009 IBM study of more than 1,000 IT and line of business decision makers around the world regarding perceptions on cloud computing. The findings revealed that although 73 percent of respondents were familiar with cloud delivery methods prior to participating in the survey, there was little consistency among the terms that respondents associated with internal or external delivery methods. Without a common vocabulary and a standardized frame of reference, it is difficult if not impossible for organizations to have a cogent discussion about cloud computing. This alone creates a barrier to developing a successful enterprise adoption strategy for cloud computing.

The rest of the paper is organized as follows. Section II presents related work, section III presents the methodology while section IV details the framework and how it was validated and lastly section V concludes this paper.

\section{RELATED WORK}

Cloud computing is at the moment in its infancy, however its adoption has great potential [9]. It has brought a new era, which has great opportunities and potential for small and medium sized enterprises and for individuals that want to increase their productivity in general [15]. At the enterprise level, cloud services became appropriate alternatives to e-mail, office software and enterprise resource planning (ERP) systems. SMEs can now gain access to highly complex IT resources freeing them the need for large infrastructural investments. Cloud computing has spread out through the main areas related to information systems and technologies, such as operating systems, application software and technological solutions for firms. SMEs are one of the most aggressive adopters of ERP packages. [5] Notes that online services are better suited for small industries whereas large enterprises face more problems in implementation because of their complex functionalities and data security concerns. Small and Medium businesses also have sufficient IT budgets to buy the bandwidth and pay as per their need and usage. The main problem faced by the SMEs when it comes to traditional ERP implementation is that their requirements are limited while the product offered always exceeds their specifications in every way. It is not possible for the traditional ERP providers to bring down their standards for the sake of the SMEs neither is it feasible for the later to upgrade for the sake of the former. Either of these if done leads to direct losses for either one party or both [5]. As the traditional in-house implementation of ERP solutions incurs high cost for the SMEs, it becomes a major constraint for them and this is where cloud computing comes in. Utilization of cloud services using smartphones is on the rise. Smartphones are giving a very clear view on one of the most important advantages of the Cloud, which is mobility. In a study, [15] observed that during the past few years, sales in smartphones surpassed sales in personal computers. This implies that this trend is giving impulse of growth and development of the cloud. Google drive and Dropbox are some of basic cloud services that are accessible through smartphones [17]. Open source Cloud Computing is one more trend that is becoming more popular. It is as a result of a huge push on this direction by different technology companies to create "powerful cloud services on open standards". One of the pioneers was Apache software foundation that developed framework used by many hi-tech companies and gives opportunity to provide a reliable analysis for both complex and structure data [16].

\section{METHODOLOGY}

This study, after critical analysis of the literature and careful evaluation of different research designs, it employed an exploratory design since investigations on models/frameworks an organization can use when it intends to adopt cloud computing are sparse and the limited number of investigating models/frameworks for adopting cloud computing means that the research in this field is young and therefore there is the need to be flexible and adaptable in this research.

\subsection{Population and Sample}

This paper employed cluster sampling technique in selecting a sample from the target population. The sampling technique was employed as it considers diversity within a target population and selects those clusters that are representative of the entire populations considering the constraints faced. While at least 10 percent of the accessible population is adequate for the sample size [13], a report by the Micro and Small Enterprise authority- Kenya of 2017, stated that there were 754 registered SMEs in Nairobi County. Random sampling technique was employed to sample individual SMEs within the clusters as it eliminated bias since each member of the target population had an equal chance or probability of being selected. From each cluster, 30 SMEs were chosen making a sample size of 120 SMEs which was adequate for the study. The statistical justification for this is a constraint on time to cover all the possible SMEs. The study targeted respondents from 
the ICT department who are in charge of administration of IT hardware and software applications, the underlying infrastructure and the various end user access tools desktops, laptops, tablets and smart phones.

\subsection{Data Collection \& Analysis}

We administered questionnaires to the targeted respondents. The objective of administering open-ended questionnaires was to find out the extent of use of computing technology in SMEs and to establish the awareness of respondents with cloud adoption frameworks. They were collected and analyzed individually and separately according to the objectives of this research. Presentation of the data was done by use of tables, charts and graphs developed by use of Microsoft excel. Only $45 \%$ of the respondents had adopted public cloud, owing to the advantages offered by public clouds. Loss of control, performance, security \& reliability, and inability to customize services were the major challenges encountered in these clouds. Out of those SMEs that had adopted, $74 \%$ had their clouds implemented by a consultant in partnership with internal team, 26\% had their clouds implemented by an external company/consultant and no SME had their internal team handle cloud implementation solely. Most respondents were not conversant with any cloud adoption frameworks. They also noted that the few choices given in the questionnaire were generic, they don't address the security issue adequately, and they don't consider externalities affecting the adoption process.

\section{FRAMEWORK DEVELOPMENT VALIDATION}

\subsection{Framework Development}

The framework was developed based on the information obtained from the literature review and the questionnaire analysis findings which were already documented. The proposed six-phase framework, which is an extension of a five-phase cloud framework by [8] describes a roadmap that indicates a clear process for cloud computing adoption. This framework has focus on the areas of trust, security, legal and compliance, as well as TOE/DOI contexts.

At a high level there will be some sort of steering committee which will comprise of key stakeholders within the SME (top-level management, ICT managers, finance controllers, procurement officers etc.). The committee will work on the business case of going to the cloud. Once the cloud migration strategy is in place, readiness of the SME and its applications to move to cloud will be assessed through pilots and prototypes. The six phases are discussed below:

Analysis: As with all projects, the initial stage is understanding users' requirements in order to determine whether the project is feasible [8]. It is at this stage that the initial requirements, feasibility, project scope, costs and initial plan will be developed. An analysis of the existing systems, applications and processes is conducted, by use of tools such as SWOT analysis, PESTLE analysis in order to develop a business case. A business case will include all key benefits which will give clear reason to move (or not) to Cloud. Thought should be given to how the existing systems strengths and opportunities can be maximized, weaknesses and threats minimized, the impact to organization culture, processes, and structure minimized, how return on investment and costs to adopting cloud computing can be managed and the usability and access to IT resources will be assured and maintained.

Planning: In this phase benchmarks for security, legal and compliance as well as technology issues identified in the analysis phase are set. The benchmarks reflect the internal organizational best practices, policies and standards to industry standards and best practices and how these can be achieved when moving to the cloud. This phase also deals with evaluating and choosing the platforms for deployment, the cloud infrastructure, technologies and best practices suitable for the organization's systems and applications, finance plan (Financing and cost management plan is developed and how costs will be managed. In preparing the adoption/roll-out plan it is important at this stage to come up with a plan for prototyping (running a pilot project) of the cloud services before full roll-out for identification of flaws and risks and how they are to be mitigated. Outsourcing strategies and vendor selection are decided upon and the benchmarks developed in this phase are used to measure vendor ability to provide service that will not affect the organization's service delivery and business.

Prototyping: Prototyping is done to provide manufacturing and assembly data, to investigate system integration issues and to develop analysis and testing strategies. By creating prototypes at a low resource cost, SMEs are able to rapidly conduct user evaluations of the cloud as well as start understanding trends in user preference at an early stage in the adoption process which allows them to make timely strategic decisions. For prototyping to be successful, it is necessary to enlist leadership and support from senior management from the outset. Obtaining such support may require one or more initial training sessions for top level officials. This phase will involve identifying the application/business processes within the enterprise which will be good candidates for testing / piloting in the Cloud. In most cases, these will be non-mission critical applications yet important ones to be able to show performance and benefits. Testing, on the other hand is a process used to identify the correctness, completeness and quality of a product [6]. Several tests such as integration and useracceptance tests can be performed on the developed prototype to refine user requirements. The prototype can either be discarded or enhanced to meet the user requirements. 


\section{PROPOSED CLOUD COMPUTING ADOPTION FRAMEWORK}

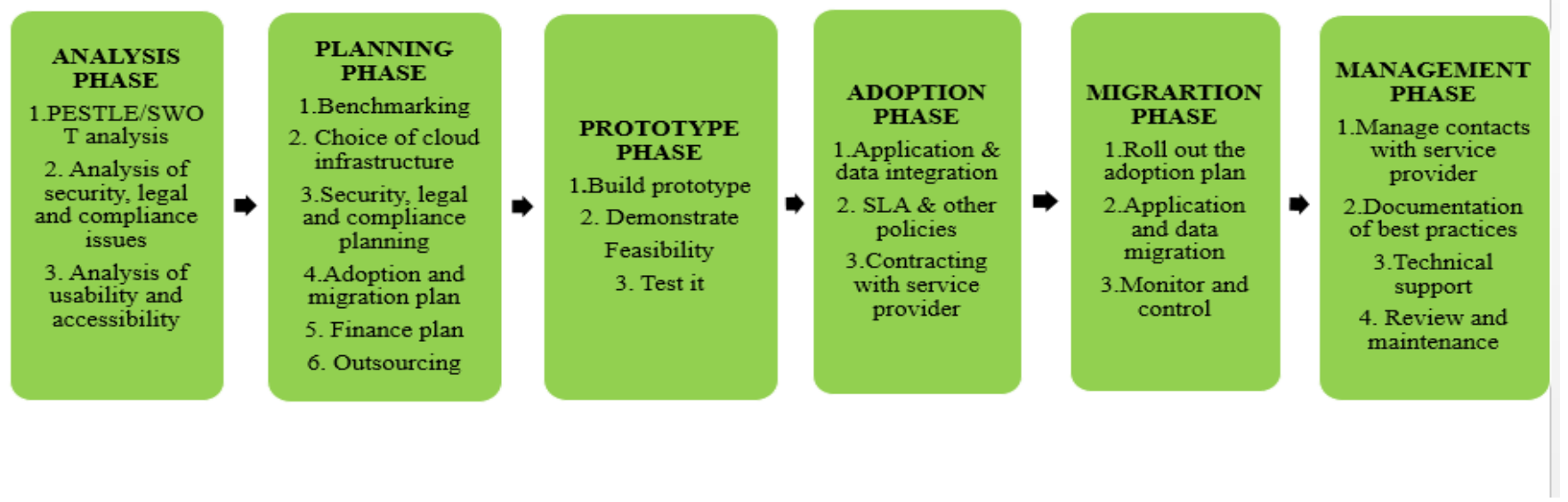

Figure 4: Proposed cloud computing adoption framework

Adoption: This is a preparation phase for the actual migration of systems and applications selected to the cloud platform and infrastructure of choice. In this phase, systems and application integration is done to ensure that the candidate applications can function with the internal applications that are not migrated to the cloud. Works on SLAs (collaboration with vendors is crucial in establishing SLA agreements and best practices to ensure compliance and trust.), customer service management, security policies, legal and compliance management and a contract with vendor is developed and signed (the contract should meet user requirements for using cloud services). At this phase, SME priorities are focused and a strategy that offers a balance between the migration costs and getting needed business value in time is worked on.

This also involves looking at all the internal and external technology and business dependencies and deriving the most optimal way from the available options.

Migration: At this phase, the refined roll-out plan can be put into practice. The migration phase ensures that application and data migration are carried out as specified in the refined roll-out plan which was developed and tested in the planning and prototyping phases, respectively. Applications and data migration is conducted. Attention should be given to application/data for migration that are more critical to the SME. Depending on the migration strategy SMEs have two options with a Cloud infrastructure - public and community (see figure 5). Within each of these options, they have the migration paths of - IaaS, SaaS or PaaS. The choice is driven by priorities such as scalability, ondemand provisioning, economics, and pay-as-you-go model and constrained by factors such as security, migration costs, existing IT investments, etc. SMEs also have an option of deploying a hybrid approach in which they can mix and match migration paths depending on business criticality and security concerns of a given application or business process. In the spectrum of Cloud offerings, the relative standardization and effectiveness increases as you go from community to public option. The community option gives benefits like ownership of data and perceived security but it might cost more than a public cloud option. In the current environment, even though the benefits of moving to Cloud computing are clear, the security and data ownership fears are making enterprises slow down on making a complete shift to the cloud - community clouds fill this gap by giving control of data and security to enterprise IT. Respondents in this research also indicated the possibility of migrating to hybrid clouds for customized services. 


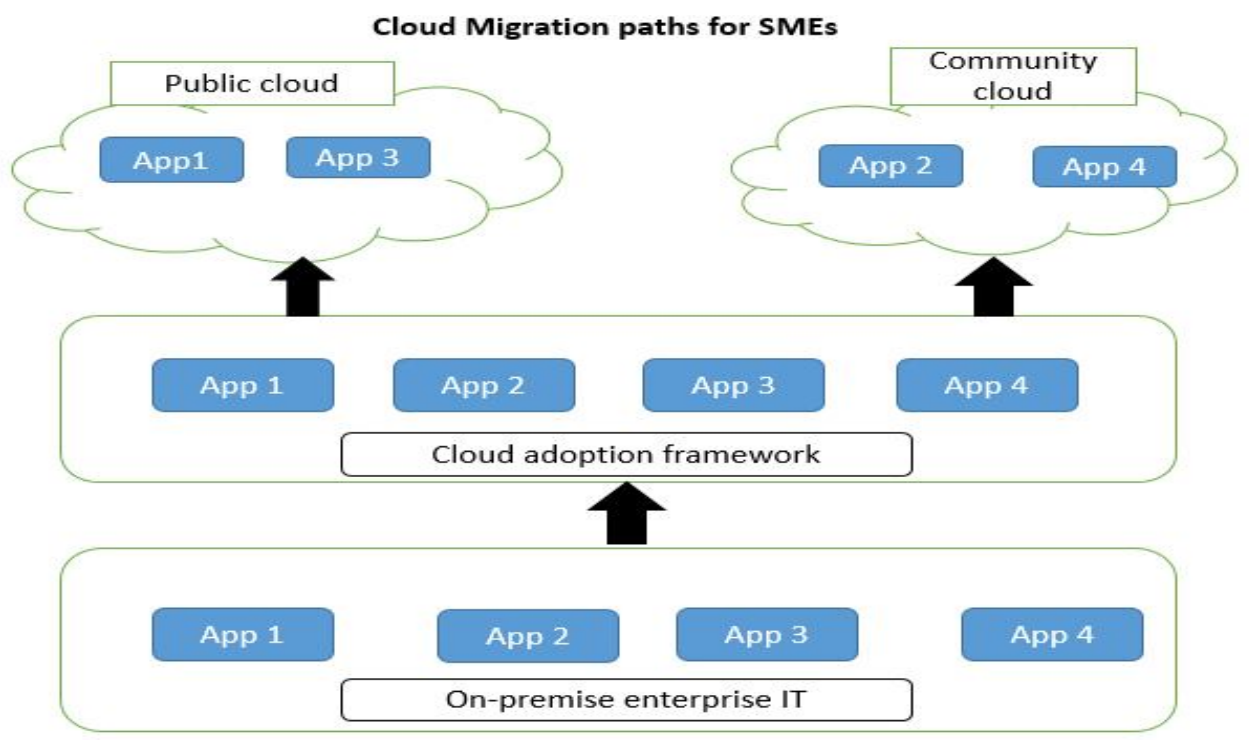

Figure 5: Cloud Migration paths for enterprises

Support and training to users during the migration process should be provided, and monitoring and control of the project should also be maintained to ensure successful migration. Finally, it is always recommended to take small group of applications/data and move them in a phased approach for better understanding and risk mitigation.

Management: The project now should be fully operational in the cloud. However contract and vendor management, testing and maintenance, user support and review should be ongoing for several months subsequent to launch. System metrics or benchmarks developed and set in phase 2 can be used as indicators of project success and should be monitored. Security standards compliance, SLAs, legal and compliance issues, IT governance best practices, service availability, throughput, scalability and cost management are desirable metrics. Best practices and lessons learnt should be documented, technical support is ensured for continual support of the systems and users. During testing, proper impact analysis (technical as well as business) should be done. Depending on the success of testing, applications/service can be customized or repackaged for easy consumption by enterprise users.

\subsection{Framework Validation}

This paper used the desirable features of a cloud computing framework that were gathered from respondents as the parameters for the evaluation. Each factor was framed as a question, which should be answered on a scale of 1 to 5.1 indicates an unfavorable response (Strongly disagree) to the question while 5 indicates a strongly favorable response (Strongly Agree). Consequently, 1 implied that the framework had not met that specific requirement while 5 implied that the framework had satisfactorily met the requirement. We then involved participants in two main focus group techniques:

Discussion. This technique was carried out to get personal guidance from an expert. We discussed, in details every phase of the proposed framework with respondents in order to identify any grey areas that needed to be revisited. Essentially, this technique was helpful in getting expert intuition to validate the framework.

Interviews. The researcher used informally in-depth interviews to investigate whether the framework addressed security, organizational and technological contexts that affect the adoption process.

Respondents confirmed and agreed that with the six phases, the framework was comprehensive, resourceful and indicated that they found the framework to be useful and an effective tool for analyzing and implementing cloud adoption projects. This was confirmed from the feedback obtained whereby 80 percent of the evaluation questions had a "strongly agree" response while 14 percent had "Agree" and only 6 percent had "Not sure" response.

\section{CONCLUSION AND FUTURE WORK}

IT has critically become an indispensable tool for the daily operations of enterprises due to its large-scale application. This research has provided a framework that will be of immeasurable help to SMEs in executing their strategy in cloud computing adoption. This research highly recommends SMEs to develop a cloud strategy and to implement the proposed framework in adopting cloud computing technology due to its desirable characteristics that can greatly improve SMEs operations. Further research looking at the effect of 
determinant contexts as highlighted in the TOE/DOI frameworks in each implementation phase is suggested or a study to measure the effects cloud computing technology has on enterprise IT systems like ERP.

\section{REFERENCES}

[1] "An Overview of the AWS Cloud Adoption Framework Version 2", 2017.

[2] "An Oracle White Paper. Oracle Consulting Cloud Services Framework”, 2011.

[3] Buyya, R., Yeo, C.S., Venugopa, S., Broberg, J., \& Brandic, I. "Cloud computing and emerging IT platforms: vision, hype, and reality for delivering computing as the 5th utility". Future Generation Computer Systems, 25, 599-616, 2013.

[4] Clarke, R. "Computing clouds on the horizon, Benefits and risks from the user's perspective". Paper presented at the 23rd Bled eConference, Bled, Slovenia, June 20-23, 2010.

[5] Dubey A. and Wagle D. "Delivering Software as a Service, the McKinsey Quarterly Web Exclusive”, 2014. [6] Gaurav Saini \& Kestina Rai. "An Analysis on Objectives, Importance and Types of Software Testing". IJCSMC, Vol. 2, Issue. 9, September 2013, pg.18-23, 2013.

[7] IBM Global Technology Services, Thought Leadership White Paper. "Defining a framework for cloud adoption", 2010.

[8] Isra M., Er. Ankur Bhardwaj, Er. Sachin Chaudhary. "An Analysis of the Existing Frameworks in Cloud Computing Adoption and Introduction to RAF". Int. Journal of Engineering Research and Applications ISSN: 2248-9622, Vol. 6, Issue 4, 2016.

[9] Kritika V., Sonal D. and Dr. Rizvi M.A. "Mobile Cloud A New Vehicle for Learning: m-Learning Its Issues and Challenges". International Journal of Science and Applied Information Technology Volume 1, No.3. 2012.
[10] Monjur A. and Mohammad A. H. "CLOUD COMPUTING AND SECURITY ISSUES IN THE CLOUD". International Journal of Network Security \& Its Applications (IJNSA), Vol.6, No.1. 2014.

[11] Montalbano, E. "General Services Administration: Cloud computing is safer than you think". Information Week, 1-5 2011.

[12] Morteza G, Jose B. A. and Daniel A-A. "Reasons for information technology adoption and sophistication within manufacturing SMEs". POMS 22nd Annual Conference: Operations management: The enabling link Reno, Nevada, U.S.A. 2011.

[13] Mugenda, D. M. and Mugenda, A. G. Research Methods Nairobi Act Press 2003.

[14] Murthy K. and Selvam R. "Security Issues and challenges in Cloud Computing". International Advanced Research Journal in Science, Engineering and Technology Vol. 2. 2015.

[15] Oliveira, T., Thomas, M., \& Espadanal, M. "Assessing the determinants of cloud computing adoption: An analysis of the manufacturing and services sectors". Information \& Management 51(5). 2014.

[16] Ryan, P. and Falvey, S. "Trust in the clouds". Computer Law and Security Reviews, 28, 513-521 2012.

[17] Shawish A. and Salama M. "Cloud Computing: Paradigms and Technologies", 2014.

[18] Tabitha K. \& David G. "Adoption and Use of Cloud Computing in Small and Medium Enterprises in Kenya".

IIMC International Information Management Corporation, 2013.

[19] Victor C., Robert J. W. \& Gary W. "Review of Cloud computing and existing Frameworks for Cloud Adoption". Advances in Cloud Computing Research, 2016.

[20] Wanjau, K. L. "The Role Of quality in growth of small and medium enterprises and economic development in Kenya", 2010. 